\title{
Translocation dynamics of a short polymer driven by an oscillating force
}

\author{
Nicola Pizzolato, ${ }^{1}$ Alessandro Fiasconaro, ${ }^{2}$ Dominique Persano Adorno, ${ }^{1,3}$ \\ and Bernardo Spagnolo 1,3, a) \\ ${ }^{1}$ Dipartimento di Fisica e Chimica, Università di Palermo and CNISM, Viale delle Scienze edificio 18, \\ I-90128 Palermo, Italy \\ ${ }^{2}$ Departamento de Física de la Materia Condensada and ICMA (CSIC-Universidad de Zaragoza), \\ 50009 Zaragoza, Spain \\ ${ }^{3}$ Group of Interdisciplinary Physics, Università di Palermo, Viale delle Scienze edificio 18, \\ I-90128 Palermo, Italy
}

(Received 14 September 2012; accepted 9 January 2013; published online 1 February 2013)

\begin{abstract}
We study the translocation dynamics of a short polymer moving in a noisy environment and driven by an oscillating force. The dynamics is numerically investigated by solving a Langevin equation in a two-dimensional domain. We consider a phenomenological cubic potential with a metastable state to model the polymer-pore interaction and the entropic free energy barrier characterizing the translocation process. The mean first translocation time of the center of inertia of polymers shows a nonmonotonic behavior, with a minimum, as a function of the number of the monomers. The dependence of the mean translocation time on the polymer chain length shows a monotonically increasing behavior for high values of the number of monomers. Moreover, the translocation time shows a minimum as a function of the frequency of the oscillating forcing field for all the polymer lengths investigated. This finding represents the evidence of the resonant activation phenomenon in the dynamics of polymer translocation, whose occurrence is maintained for different values of the noise intensity. (C) 2013 American Institute of Physics. [http://dx.doi.org/10.1063/1.4789016]
\end{abstract}

\section{INTRODUCTION}

The transport of molecules across membranes and the translocation of a polymer through a nanopore represent very important processes in the science of the living systems. Diffusion of molecules from a region of higher concentration to one of lower concentration, as a result of thermal motion, is the mechanism of movement of oxygen, nutrients, and other molecules across capillary walls and membranes. The separation of electric charges across a membrane can act independently of, or in conjunction with, or in opposition to, the force generated by concentration differences. As a consequence, this separation can influence the movement of ions across the membrane. In cell environment, DNA and RNA translocate across nuclear pores and many proteins work on the bases of their ability to go beyond a potential barrier. Recent studies on anticancer therapy investigate the efficacy of targeted treatments. These are based on a drug delivery mechanism that crucially depends on the translocation time of the chemotherapeutic molecule carriers. ${ }^{1,2}$ The study of the transport dynamics of macromolecules across a nanopore is also important in the development of technologies that can be applied to improve DNA or RNA molecule sequencing techniques ${ }^{3,4}$ or to develop nanostructure-based electrical biosensors. ${ }^{5,6}$

First experiments on polymer translocation were carried out by detecting the passage of DNA molecules across an $\alpha$-hemolysin ( $\alpha$-HL) protein channel, by measuring the reduction of the electrolyte ion current. ${ }^{7-10}$ In this way, a linear relationship of the most probable crossing time $\tau_{p}$ with

a)Electronic mail: bernardo.spagnolo@unipa.it. the molecule length was established. Moreover, $\tau_{p}$ scales as the inverse square of the temperature and the dynamics of biopolymer translocation across an $\alpha-\mathrm{HL}$ channel is found to be governed by pore-molecule interactions. On the other hand, interesting experiments on the transport dynamics of DNA molecules driven inside an entropic trap have shown longer crossing times for shorter DNA molecules. This has suggested the existence of a quasi-equilibrium state of the polymer during the translocation dynamics. ${ }^{11,12}$ More recent experiments have shown that the application of an ac voltage, to drive the translocation process of DNA molecules through a nanopore, plays a significant role in the DNA-nanopore interaction. This has provided new insights into the DNA conformations. ${ }^{13-19}$ Moreover, the living cells give rise to a fluctuating environment. As a consequence, the polymers are subject to time dependent driving forces, which drive the biological system far away from equilibrium. ${ }^{18,19}$

Several different theoretical models try to predict the complex translocation features. This, under different conditions of geometrical confinements of the molecule, pore interaction, inertia ${ }^{20-40}$ and/or different time dependent driving mechanisms. ${ }^{41-47}$

In spite of the experimental and theoretical work done, the complicated boundary conditions, and out of equilibrium translocation dynamics due to the biological environment, make the problem of biopolymer translocation still far from being completely understood. In this framework, a detailed description of the transport dynamics of short polymers driven by an oscillating driving field is missing. In this work we investigate the translocation dynamics of a short polymer driven by an oscillating force, in the presence of a metastable 
state. Specifically, we analyze the translocation time of a polymer through a potential barrier with a metastable state. This phenomenological potential profile models the polymerpore interaction and the entropic free energy barrier characterizing the translocation process. Moreover, the time dependent driving force mimics the effect of the fluctuating environment, which drives the polymer out of equilibrium during the translocation process. The role of the polymer chain length and frequency of the oscillating forcing field on the mean first translocation time (MFTT) is investigated.

We find a minimum of the MFTT of the molecule center of mass as a function of the frequency of the force. This nonmonotonic behavior confirms the occurrence of the resonant activation (RA) phenomenon in polymer translocation. This phenomenon is characterized by the presence of a minimum in the mean time as a function of the frequency of the force, spent by a single Brownian particle in surmounting a potential barrier. ${ }^{48-54}$ In other words, the MFTT that the chain molecule takes to cross the potential barrier can be increased, or reduced, by suitably tuning the oscillating field. We also find a nonmonotonic behavior, characterized by the presence of a minimum, of the MFTT as a function of the molecule chain length. Moreover, the mean first translocation times increase monotonically with polymer chain length for high values of the number of monomers $N$. The paper is organized as follows. In Sec. II we present our polymer chain model and give the details of the molecular dynamics simulation process. Results are reported in Sec. III and conclusions are given in Sec. IV.

\section{POLYMER DYNAMICS MODEL}

In this work the polymer is modeled by a semi-flexible linear chain of $N$ beads connected by harmonic springs. ${ }^{55}$ Both excluded volume effect and van der Waals interactions between all beads are kept into account by means of a Lennard-Jones (LJ) potential. With the aim to confer a suitable stiffness to the chain, a bending recoil torque is included in the model, with a rest angle $\theta_{0}=0$ between two consecutive bonds. To first order, we neglect any hydrodynamic effect induced by the polymer motion in the solvent. Such approximation implies that our model is very appropriate for polymeric melts, ${ }^{56}$ but cannot be used to simulate the behavior of chain molecules in dilute polymeric solutions. The total potential energy of the modeled chain molecule is $U=U_{\mathrm{Har}}$ $+U_{\mathrm{Bend}}+U_{\mathrm{LJ}}$ with

$$
\begin{gathered}
U_{\mathrm{Har}}=\sum_{i=1}^{N-1} \frac{1}{2} K_{\mathrm{r}}\left(r_{i, i+1}-d\right)^{2}, \\
U_{\mathrm{Bend}}=\sum_{i=2}^{N-1} \frac{1}{2} K_{\theta}\left(\theta_{i-1, i+1}-\theta_{0}\right)^{2}, \\
U_{\mathrm{LJ}}=4 \epsilon_{\mathrm{LJ}} \sum_{i, j(i \neq j)}\left[\left(\frac{\sigma}{r_{i j}}\right)^{12}-\left(\frac{\sigma}{r_{i j}}\right)^{6}\right],
\end{gathered}
$$

where $K_{\mathrm{r}}$ is the elastic constant, $r_{i j}$ is the distance $\sqrt{\left(x_{i}-x_{j}\right)^{2}+\left(y_{i}-y_{j}\right)^{2}}$ between particles $i$ and $j, d$ is the equilibrium distance between adjacent monomers, $K_{\theta}$ is the bending modulus, $\theta_{i-1, i+1}$ is the angle between the bonds of the polymer having the bead $i$ as central monomer, $\epsilon_{\mathrm{LJ}}$ the LJ energy depth and $\sigma$ the monomer diameter. The effect of temperature fluctuations on the dynamics of a chain polymer escaping from a metastable state is studied in a twodimensional (2D) domain. The 2D model for the chain, and the corresponding dynamics, is a first step towards a full 3D description. It is fairly used in literature (see, for example, Ref. 46) and gives the possibility of introducing the bending magnitude into the model. That way it allows to include more realistic effects on the chain dynamics, which are not present in a mere $1 \mathrm{D}$ description.

The translocation dynamics of a polymer through a narrow pore is characterized by the presence of an entropic barrier. ${ }^{35,57-62}$ Indeed the predictions of the entropic barrier model have been observed in many single-molecule electrophysiology experiments. ${ }^{7,10,63-65}$ Here, the polymer translocation is modeled as a stochastic process of diffusion in the presence of a phenomenological potential barrier having the form

$$
U_{\text {Ext }}(x)=a x^{2}-b x^{3} \text {, }
$$

with parameters $a=3 \times 10^{-3}$ and $b=2 \times 10^{-4}$, as already adopted in Ref. 31. A three-dimensional view of $U_{\text {Ext }}$ is plotted in Fig. 1.

The phenomenological potential profile with a metastable state of Eq. (4) is used to model the polymer-pore

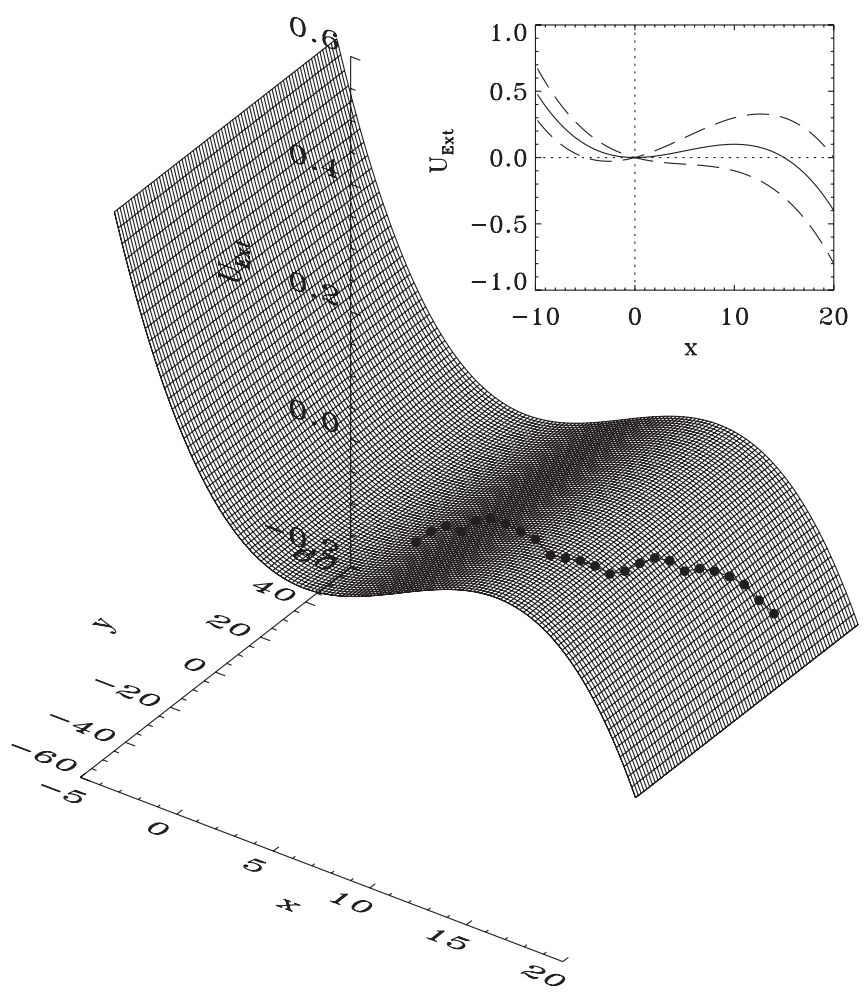

FIG. 1. 3D-view of the phenomenological potential energy $U_{\text {Ext }}$, which is included in our system to simulate the presence of a barrier to be surmounted by the polymer during the translocation dynamics. The inset shows the projection of $U_{\mathrm{Ext}}$ on the $z-x$ plane (solid line); dashed lines indicate the maximum and the minimum shape of the potential barrier caused by the presence of the oscillating forcing field. 
interaction and the entropic free energy barrier. The translocation process of a polymer through an ion channel involves the transport of a polymer from a region outside the channel, in which many chain conformations are allowed, and the channel, where the number of allowed conformations is strongly reduced. As a consequence, the polymer chain is transported across an entropic free energy barrier. In other words, the polymer chain entropy decreases and the corresponding free energy increases $(\mathrm{F}=\mathrm{E}-\mathrm{TS}$, with $\mathrm{E}$ the interaction energy between monomers and the surrounding solvent molecules, $\mathrm{T}$ the absolute temperature, and $\mathrm{S}$ the entropy). The so-called entropic barrier is indeed a freeenergy barrier because additional enthalpic contributions to the free energy can arise from the interactions between the polymer and the pore. In the final stage, the polymer chain reaches a region with low free energy. By summarizing, the translocation process could be described as the kinetic evolution of a metastable state into an equilibrium state separated by a free-energy barrier. ${ }^{62}$ Moreover attractive polymerpore interactions contribute to create the free energy well on the left of the free energy barrier. $^{46,62}$ This motivates our choice of using the phenomenological potential profile described by Eq. (4). Therefore, the metastable state represents a pretransitional well, and the polymer translocation dynamics becomes a barrier crossing event activated by thermal fluctuations, described as a Gaussian uncorrelated noise. ${ }^{11,12,19,24,27,28,31,35,41,46,62}$

The diffusion of the $i$ th monomer of the chain molecule along the free energy landscape provided by $U_{\text {Ext }}(x)$ and subject to the total potential energy $U$ (see Eqs. (1)-(3)) is described by the following overdamped Langevin equations:

$$
\begin{aligned}
\frac{d x_{i}}{d t} & =-\frac{\partial U}{\partial x_{i}}-\frac{\partial U_{\mathrm{Ext}}\left(x_{i}\right)}{\partial x_{i}}+\sqrt{D} \xi_{\mathrm{x}}+A \cos (\omega t+\phi), \\
\frac{d y_{i}}{d t} & =-\frac{\partial U}{\partial y_{i}}+\sqrt{D} \xi_{\mathrm{y}},
\end{aligned}
$$

where $U$ is the interaction potential between the $i$ th and $j$ th beads, $\xi_{x}$ and $\xi_{y}$ are white Gaussian noise modeling the temperature fluctuations, with the usual statistical properties, namely $\left\langle\xi_{k}(t)\right\rangle=0$ and $\left\langle\xi_{k}(t) \xi_{l}(t+\tau)\right\rangle=\delta_{(k, l)} \delta(\tau)$ for $(k, l$ $=x, y) . A$ and $\omega$ are, respectively, the amplitude and the angular frequency of the forcing field and $\phi$ is a randomly chosen initial phase. In our simulations, the time $t$ is scaled with the friction parameter $\gamma$ as $t=t_{r} / \gamma$, where $t_{r}$ is the real time of the process. The standard Lennard-Jones time scale is $\tau_{\mathrm{LJ}}$ $=\left(m \sigma^{2} / \epsilon_{\mathrm{LJ}}\right)^{1 / 2}$, where $m$ is the mass of the monomer. A bead of a single-stranded DNA is formed approximately by three nucleotide bases and then $\sigma \sim 1.5 \mathrm{~nm}$ and $m \approx 900$ amu. ${ }^{26}$ Orders of magnitude of the quantities involved in the process are nanometers for the characteristic lengths of the system, polymer, and barrier extension, and microseconds for the time domain.

A different number of numerical simulations, ranging from $2 \times 10^{3}$ for longer polymers up to $20 \times 10^{3}$ for shorter chains, has been performed for several values of the frequency of the forcing field and three values of the noise intensity $D$, namely, $D=0.25,0.5,1$. The values of the potential energy parameters are: $K_{\mathrm{r}}=K_{\theta}=20, \epsilon_{\mathrm{LJ}}=0.1, \sigma=3$, and $d=5$, in arbitrary units (AU). The amplitude of the forcing field is $A$
$=2 \times 10^{-2}$ in $\mathrm{AU}$. The number of monomers $N$ ranges from 15 to 60 . The initial spatial distribution of the polymer is with all monomers at the same coordinate $x_{0}=0$, corresponding to the local minimum of the potential energy of the barrier. Every simulation stops when the $x$ coordinate of the center of mass of the chain reaches the final position at $x_{f}=15$. This is because, we calculate the mean first translocation time and we do not consider events that bring back the polymer chain, after crossing the absorbing boundary at $x_{f}$.

\section{RESULTS AND DISCUSSION}

The MFTT shows three different translocation regimes as a function of the frequency of the forcing field (Fig. 2). In the low frequency domain $\left(\omega<10^{-3}\right)$, the period of the forcing field oscillations is very long with respect to the typical values of the mean crossing time of the chain molecule. In this regime the MFTT is equal to the average of the crossing times over the upper and lower configurations of the barrier, and the slower processes critically affect the value of the mean crossing time. In other words, in the $\omega \rightarrow 0$ limit the oscillation period is slower than any characteristic timescale of the problem. The time dependent periodic term in the first of Eqs. (5) could then be replaced by a constant term $a=A$ $\cos (\phi)$, leading to a MFTT of $T(a)$. In that limit the MFTT can be expressed as

$$
\langle T\rangle=\int_{-A}^{A} T(a) p(a) d a,
$$

where $p(a)$ is the distribution of the a's.

In the high frequency domain $\left(\omega>10^{-1}\right)$, a saturation of the translocation time is obtained. In this case, very rapid oscillations of the potential make the molecule chain "feel" the average potential barrier and, therefore, the MFTT becomes equal to that obtained without any additional periodic driving.

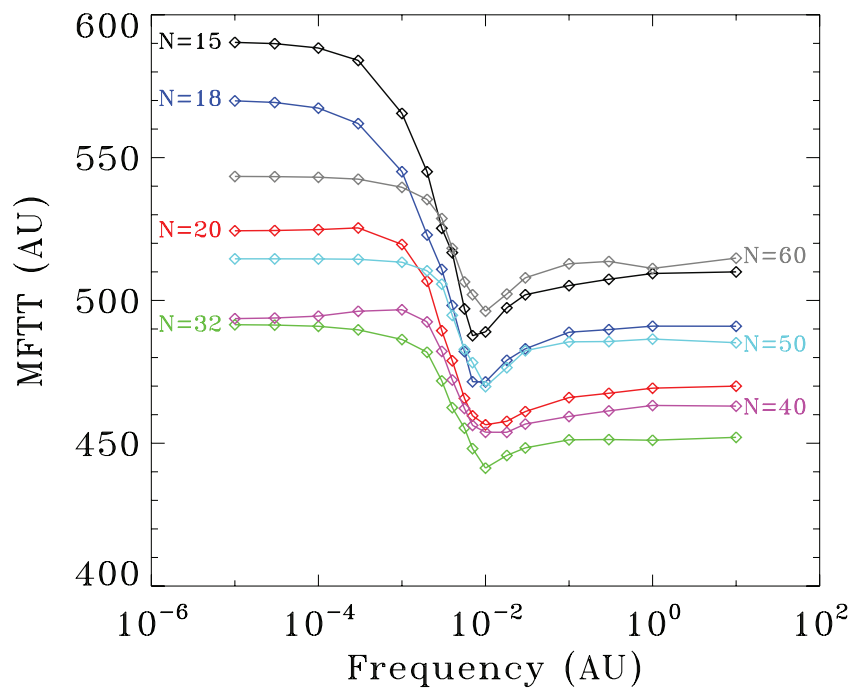

FIG. 2. MFTT vs. frequency of the forcing field for seven different values of the number of monomers, namely, $N=15,18,20,32,40,50,60$. The noise intensity is $D=1.0$. The values of the potential energy parameters are: $K_{\mathrm{r}}$ $=K_{\theta}=20, \epsilon_{\mathrm{LJ}}=0.1, \sigma=3$, and $d=5$, in arbitrary units (AU). The amplitude of the forcing field is $A=2 \times 10^{-2}$ (AU). 
For intermediate frequencies $\left(10^{-3}<\omega<10^{-1}\right)$, that is in the "resonant activation" regime, the crossing event is strongly correlated with the potential oscillations and the MFTT vs. $\omega$ exhibits a minimum at a resonant oscillation rate. This frequency region corresponds to periods of oscillation of the same order of magnitude of the mean time that the polymer takes to cross a static barrier in the lowest configuration of the oscillating potential. ${ }^{52-54}$ In other words, the potential remains around its lowest configuration for enough time to allow the polymer to exit and, even in the case of an initially high or intermediate value of the height of the barrier, the potential can turn into lower configurations within a sufficiently short time lag to facilitate the translocation process. The period of the oscillation is in between the mean first translocation times for $+A$ and $-A$. Translocation time depends exponentially on $A$, and the MFTT is dominated by $T(-A)$, and that is what causes the minimum. To evaluate the resonant activation minimum the average translocation rate should be taken, i.e.,

$$
\langle k\rangle=\int_{-A}^{A} k(a) p(a) d a,
$$

where $k(a)=1 / T(a)$ and the ultimate location of the minimum is then at $T_{\text {res.act. }}=1 /\langle k\rangle$.

This is the RA phenomenon, firstly studied on a Brownian particle escape dynamics, ${ }^{48-54}$ and found in polymer dynamics by Pizzolato et al. ${ }^{41}$ and with a different approach by Fiasconaro et al. ${ }^{42}$ and Ikonen et al. ${ }^{46}$ The chain molecule, driven by a periodic field oscillating at a period comparable with the characteristic time of the crossing dynamics, reaches the resonant regime that accelerates the translocation process.

The behavior of the MFTT vs. the frequency of the driving field has been investigated with polymers of different lengths. In particular, in Fig. 2 we show the translocation times of polymers having the number of monomers equal to $N=15,18,20,32,40,50,60$. The first result is that the frequency at which the resonant activation occurs, corresponding to the minimum of MFTT in Fig. 2, appears to be almost independent on the polymer length. This fact confirms that, in the studied range of the molecule lengths, the resonance phenomenon is more properly related to the constructive interplay between the periodic forcing field and the characteristic translocation dynamics of the chain.

The second result is shown in Fig. 3, in which we show the nonlinear behavior of the MFTT with the length of the polymer, driven by a forcing field oscillating at the characteristic frequencies of the three crossing regimes, namely, $\omega$ $=0.0001,0.01,1.0$. In particular, we find a common decreasing trend of MFTTs for increasing chain lengths until $N \simeq 27$. At low number of monomers, the short polymer chain "feels" all the potential profile and can be trapped in the metastable state, giving rise to a high MFTT. For increasing length of the chain, when this length becomes comparable with the size of the potential barrier along the $\mathrm{x}$ axis, because of the "independent searching" of the two ends of the polymer chain, it becomes more likely that the center of mass of polymer chain overcomes the potential barrier and reaches $x_{f}$. Therefore, the MFTT decreases. This corresponds to the minimum of MFTT in Fig. 3. Specifically, for crossing events driven by the po-

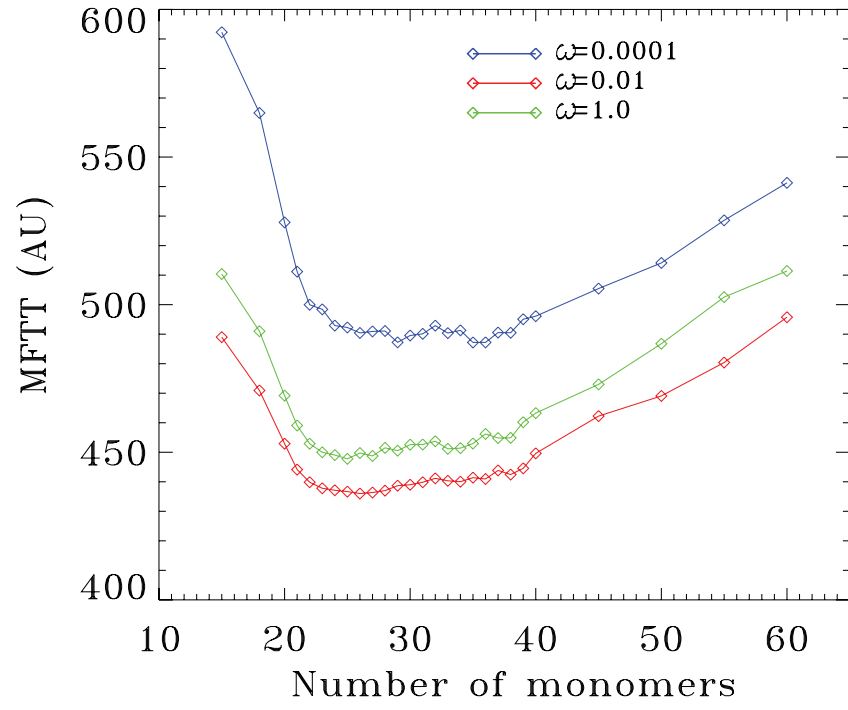

FIG. 3. Mean first translocation time (MFTT) as a function of the number of monomers for three different values of the angular frequency of the forcing field, namely, $\omega=0.0001,0.01,0.1$. For polymer chain length $N>40$, a monotonic increasing behavior of MFTT vs $N$ is observed. All other parameter values are the same as those of Fig. 2.

tential oscillating at $\omega=0.0001$, we find a plateau between $N \simeq 27$ and $N \simeq 38$. For greater lengths of polymer chain many transitions from unfolded to folded configurations occur, giving rise to a monotonic increase of the average translocation time. At the lower frequency $\omega=0.0001$, we find longer MFTTs for $N>40$. For both $\omega=0.01$ and $\omega=1.0$, an increasing trend of the mean translocation time starts with polymers having $N \simeq 30$.

The nonmonotonic behavior of MFTT vs $N$, shown in Fig. 3, is in qualitative agreement with similar findings reported in previous investigations based on different theoretical models. ${ }^{24,61}$ Here we find the same non-monotonic behavior with a minimum, located in the same range of values of polymer chain length, ${ }^{24}$ but in the presence of a periodically driving field. This time-dependent force is used to model in a realistic way the driven translocation dynamics which is inherently an out of equilibrium process. ${ }^{34,46}$ We note that the nonmonotonic dependence of the MFTT on the polymer length, characterized by a minimum, is a general peculiarity of the translocation dynamics, both in the case of constant driving force ${ }^{24,61}$ and time-dependent one (this work).

For polymer length $N>40$, independently on the frequency of the oscillating potential, the MFTT grows monotonically with the molecule length, confirming the experimental findings reported in Refs. 7-9. In other words, by increasing the polymer length, a trapping phenomenon within the metastable state occurs for which longer polymer chains take longer times to cross the potential barrier. The translocation process is slowing down.

The different dynamical regimes of translocation are further investigated by studying how the standard deviation (SD) of the first translocation time (FTT) changes as a function of the frequency of the driving field (Fig. 4). For each molecule length, we find two saturation values of the SD, characterizing the low and high frequency domain. We note that, the SD 


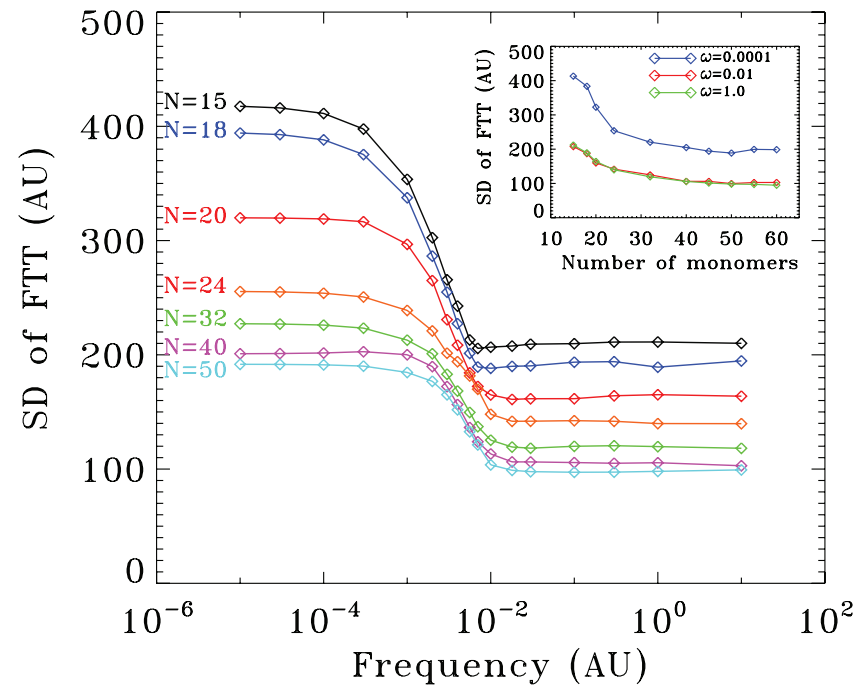

FIG. 4. Standard deviation (SD) of the first translocation time (FTT) as a function of the frequency of the oscillating field, for the same values of polymer length plotted in Fig. 2, except for $N=60$, whose curve essentially overlaps that with $N=50$ and, for this reason, it was not plotted, and $N$ $=24$ which instead was added. (Inset) The SD of FTT versus the number of monomers for three different values of the frequency of the forcing field, namely, $\omega=0.0001,0.1,1$. All other parameter values are the same as those of Fig. 2.

at high frequencies is reduced by nearly a factor two with respect to the corresponding value at low frequency. In the inset of Fig. 4 it is shown the SD of FTT versus the number of monomers, for three different values of the frequency of the forcing field, namely, $\omega=0.0001,0.1,1$.

A transition between these two regimes is found within the range $10^{-3}<\omega<10^{-2}$. For any fixed frequency, the SD always decreases with increasing the number of monomers.

In order to clarify the SD behavior of the FTT, and to investigate the details of the translocation dynamics of polymers driven by a periodic field, we have calculated the probability density function (PDF) of the FTTs. The PDFs are calculated for three values of the driving frequency, characterizing the different dynamical regimes (low, intermediate or resonant, and high frequency), and for three values of the polymer length, namely, for $N=15,30$, and 60 . In the following Fig. 5 each panel shows three PDFs, each one characterized by a specific value of the number of monomers. In the low frequency range (Fig. 5(a)) long tails in the PDFs are found, indicating that the polymer translocation in this regime is strongly affected by the presence of a forcing field, which modifies the height of the barrier over very long times. This means that, a relevant fraction of the simulated translocations takes place with the barrier in its upper configurations, resulting in longer crossing times. In the resonant activation regime (Fig. 5(b)), the PDFs do not present the long tails at very high crossing times, observed in Fig. 5(a), and the MFTT reduces its value. For $N=15$, the PDF shows an interesting hump that could suggest the presence of multiple characteristic times of translocation, probably caused by the progressive change of the height of the barrier. In the high frequency regime (Fig. 5(c)), the PDFs show the characteristic feature of the static potential case, ${ }^{66}$ and the average escape time is equal to the crossing time over the average barrier. ${ }^{52-54}$
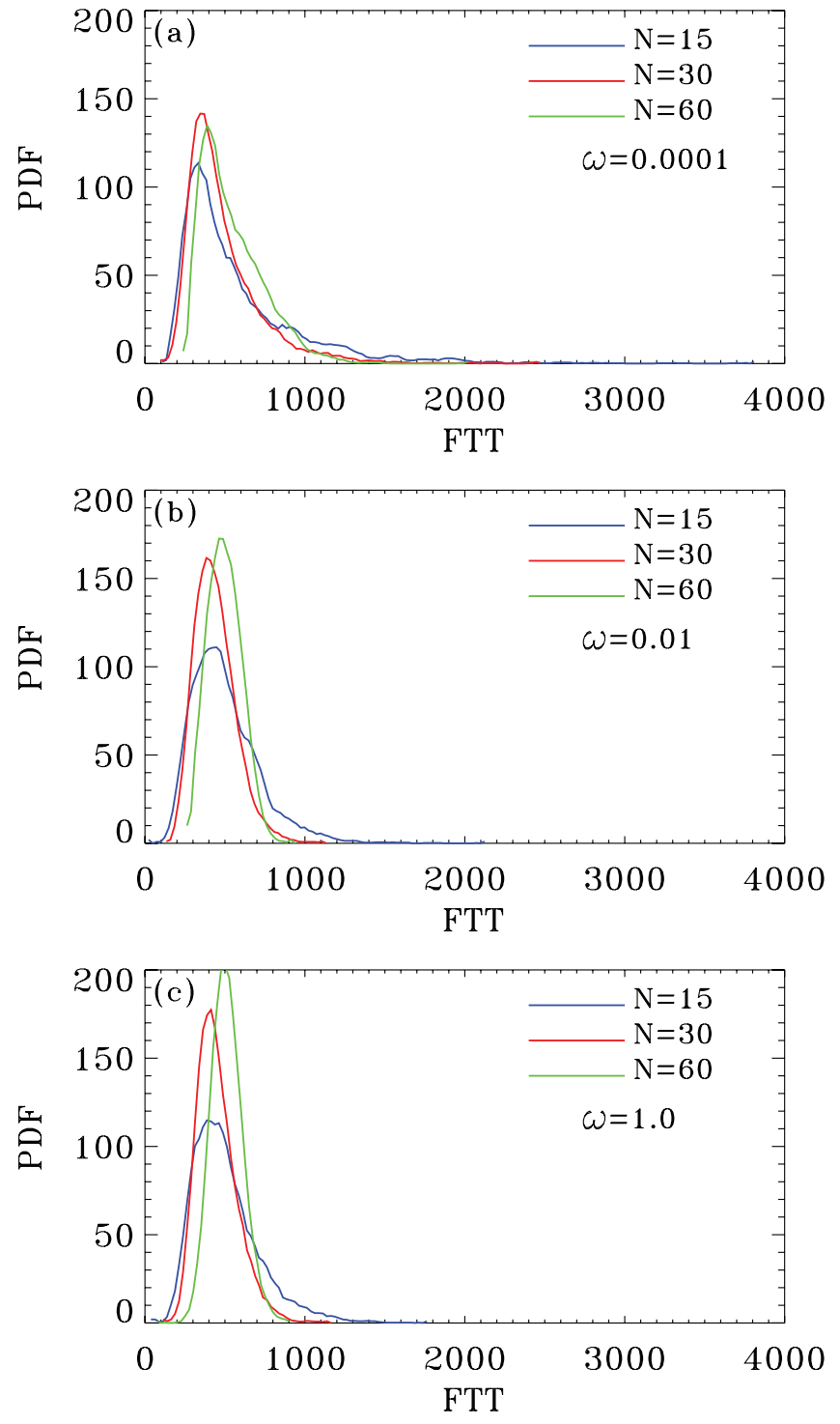

FIG. 5. Probability density function (PDF) of the first translocation time (FTT). Each panel shows three PDFs, each one characterized by a specific value of the number of monomers, namely, $N=15,30,60$. The three panels differs for the frequency of the forcing field: (a) low frequency domain $(\omega=0.0001)$; (b) resonant activation region $(\omega=0.01)$; (c) high frequency domain $(\omega=1.0)$. All other parameter values are the same as those of Fig. 2.

For all the three investigated frequencies, longer polymer chains show PDFs with shorter tails, suggesting that the dynamics of translocation takes an advantage from internal recoil torques of the chain molecule. For $N=30$ and $N=60$, the PDFs assume a narrower shape with the increase of the frequency, justifying the behavior of the SD shown in Fig. 4.

This finding means that, even if short polymers may translocate with an average crossing time equal to that experienced by longer polymers, the dynamics of the two crossing events is completely different. In fact, longer chain molecules take almost the same time to surmount the barrier, while shorter polymers have wider distributions of the translocation time.

In Fig. 6 we show how the thermal noise intensity $D$ affects the mean crossing time of polymers with constant length $(N=30)$, as a function of the frequency of the 


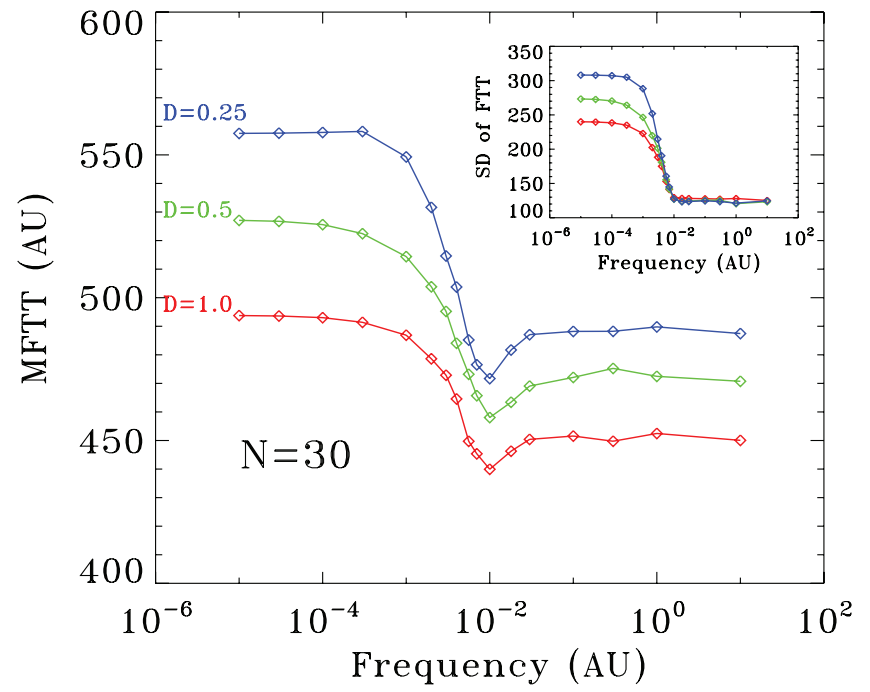

FIG. 6. Mean first translocation time of polymers having constant length $(N$ $=30$ ) as a function of the frequency of the oscillating field, for three different values of the noise intensity, namely $D=0.25,0.5,1.0$. The inset shows the standard deviation (SD) of the FTTs. All other parameter values are the same as those of Fig. 2.

oscillating potential. We have investigated the translocation process for three values of the thermal noise intensity $D$, namely, $D=0.25,0.5,1.0$. Longer mean crossing times are found at lower levels of noise. These findings are in qualitative agreement with the experiments of DNA molecules, driven through an $\alpha$-HL protein channel at different temperatures. ${ }^{10}$ However, in our calculations the rescaled absolute temperature $D$ is varied by a factor 4 (see Fig. 6), while in biological experiments the temperature dependence of the most probable translocation duration is varied by a factor $\approx 2.7$ (see Fig. 7 of Ref. 10). Our results show that, the thermal noise intensity is able to speed up or slow down the translocation process, without significantly affecting the resonant activation behavior described in Fig. 2. The inset of Fig. 6 shows the SD of the FTTs for these molecules as a function of $\omega$. We find that the SD depends on the noise intensity only in the low frequency region, while an almost equal value is observed at higher frequencies. With the increase of the noise intensity, the number of crossing events with very long times is gradually lowered, causing the observed reduction of the MFTTs and explaining the behavior of SD at low frequencies. In the high frequency domain, that is in the $\omega \rightarrow \infty$ limit, the polymer "feels" a barrier with the average height and the crossing events with very long times (related to the translocation through higher barrier configurations) are absent. In other words, the modulation of the barrier is no longer effectively contributing to the standard deviation. Hence, the increase of thermal noise intensity reduces the MFTTs leaving unchanged the amplitude of the SD.

\section{CONCLUSIONS}

In this work we investigate the influence of a phenomenological oscillating potential on the translocation dynamics of polymers, with different lengths, embedded in a noisy environment. We simulate the translocation process by let- ting the polymer cross a potential barrier starting from a metastable state, in the presence of thermal fluctuations. The time-dependent driving force, which may originate from the cellular environment, drives the polymer out of equilibrium during the translocation dynamics. We find the resonant activation phenomenon in polymer crossing dynamics. The mean translocation time, as a function of the frequency of the driving force, shows a nonmonotonic behavior, characterized by the presence of a minimum at a specific frequency, which is almost independent on the molecule chain length. Moreover, we find a nonlinear behavior of the MFTT also with the polymer length. Independently on the frequency of the oscillating potential, we find an initial decreasing trend of MFTTs for increasing chain lengths until $N \simeq 27$. For longer polymers, a monotonic increasing behavior of the MFTT with the molecule length is observed, in agreement with experimental findings.

Short polymers may translocate with an average crossing time almost equal to that taken by longer polymers. Nevertheless, a detailed analysis of the FTT PDFs shows that the dynamics of the two crossing events is completely different. In fact, all longer chain molecules take almost the same time to surmount the barrier, while shorter polymers have wider distributions of the translocation time.

Shorter mean crossing times are found at higher levels of the noise amplitude, confirming experimental findings on DNA molecule translocation at different temperatures. Moreover, our results show that the resonant activation phenomenon is not significantly affected by a change of the thermal noise intensity.

Time dependent driving forces can play a fundamental role in biopolymer translocation. A forcing periodic field, jointly with the temperature of the system, can be able to speed up or slow down the polymer translocation. In this view, the oscillating field constitutes a tuning mechanism to select a suitable translocation time of the polymer. This feature can be of fundamental importance for all those experiments on cell metabolism, DNA-RNA sorting and sequencing, and drug delivery mechanism in anti-cancer therapy. Finally, a biopolymer can use the environmental nonequilibrium fluctuations to optimize self-organizing processes and control transcription.

\section{ACKNOWLEDGMENTS}

This work was partially supported by MIUR and partially by the Spanish DGICYT Project No. FIS2011-25167, co-financed by FEDER funds.

${ }^{1}$ C. F. Higgins, Nature (London) 446, 749 (2007)

${ }^{2}$ S. Halwachs, I. Schäfer, P. Seibel, and W. Honscha, Leukemia 23, 1087 (2009)

${ }^{3}$ M. Rhee and M. A. Burns, Trends Biotechnol. 24, 580 (2006).

${ }^{4}$ J. T. Mannion, C. H. Reccius, J. D. Cross, and H. G. Craighead, Biophys. J. 90, 4538 (2006).

${ }^{5}$ V. B. Sundaresan and D. J. Leo, Sens. Actuators B 131, 384 (2008).

${ }^{6}$ Z. S. Siwy and M. Davenport, Nat. Nanotechnol. 5, 174 (2010).

${ }^{7}$ J. J. Kasianowicz, E. Brandin, D. Branton, and D. W. Deamer, Proc. Natl. Acad. Sci. U.S.A. 93, 13770 (1996).

${ }^{8}$ M. Akeson, D. Branton, J. J. Kasianowicz, E. Brandin, and D. W. Deamer, Biophys. J. 77, 3227 (1999).

${ }^{9}$ A. Meller and D. Branton, Electrophoresis 23, 2583 (2002). 
${ }^{10}$ A. Meller, L. Nivon, E. Brandin, J. A. Golovchenko, and D. Branton, Proc. Natl. Acad. Sci. U.S.A. 97, 1079 (2000).

${ }^{11}$ J. Han, S. W. Turner, and H. G. Craighead, Phys. Rev. Lett. 83, 1688 (1999).

${ }^{12}$ J. Han and H. G. Craighead, Science 288, 1026 (2000).

${ }^{13}$ J. Deng, K. H. Schoenbach, E. S. Buescher, P. S. Hair, P. M. Fox, and S. J. Beebe, Biophys. J. 84, 2709 (2003).

${ }^{14}$ P. T. Vernier, Y. Sun, L. Marcu, C. M. Craft, and M. A. Gundersen, Biophys. J. 86, 4040 (2004).

${ }^{15}$ G. Sigalov, J. Comer, G. Timp, and A. Aksimentiev, Nano Lett. 8, 56 (2008).

${ }^{16}$ D. K. Lathrop, G. A. Barrall, E. N. Ervin, M. G. Keehan, M. A. Krupka, R. Kawano, H. S. White, and A. H. Hibbs, Biophys. J. 96, 647a (2009).

${ }^{17}$ A. Nikolaev and M. Gracheva, Biophys. J. 96, 649a (2009).

${ }^{18}$ D. K. Lathrop, E. N. Ervin, G. A. Barrall, M. G. Keehan, R. Kawano, M. A. Krupka, H. S. White, and A. H. Hibbs, J. Am. Chem. Soc. 132, 1878 (2010).

${ }^{19}$ Y. F. Chen, J. N. Milstein, and J.-C. Meiners, Phys. Rev. Lett. 104, 258103 (2010).

${ }^{20}$ P. J. Park and W. Sung, Int. J. Bifurcation Chaos 8, 927 (1998).

${ }^{21}$ D. K. Lubensky and D. R. Nelson, Biophys. J. 77, 1824 (1999).

${ }^{22}$ F. Tessler and G. W. Slater, Appl. Phys. A 75, 285 (2002).

${ }^{23}$ A. J. Storm, J. Chen, H. Zandbergen, and C. Dekker, Phys. Rev. E 71, 051903 (2005)

${ }^{24}$ K. F. Luo, T. Ala-Nissila, S. C. Ying, and A. Bhattacharya, Phys. Rev. Lett. 99, 148102 (2007).

${ }^{25}$ C. Forrey and M. Muthukumar, J. Chem. Phys. 127, 015102 (2007).

${ }^{26}$ K. F. Luo, T. Ala-Nissila, S. C. Ying, and A. Bhattacharya, Phys. Rev. Lett. 100, 058101 (2008).

${ }^{27}$ K. Luo, S. T. T. Ollila, I. Huopaniemi, T. Ala-Nissila, P. Pomorski, M. Karttunen, S.-C. Ying, and A. Bhattacharya, Phys. Rev. E 78, 050901(R) (2008).

${ }^{28}$ N. Pizzolato, A. Fiasconaro, and B. Spagnolo, Int. J. Bifurcation Chaos 18, 2871 (2008)

${ }^{29}$ D. Panja and G. T. Barkema, Biophys. J. 94, 1630 (2008).

${ }^{30}$ M. Wanunu, J. Sutin, B. McNally, A. Chow, and A. Meller, Biophys. J. 95, 4716 (2008).

${ }^{31}$ N. Pizzolato, A. Fiasconaro, and B. Spagnolo, J. Stat. Mech.: Theory Exp. (2009) P01011.

${ }^{32}$ K. F. Luo, R. Metzler, T. Ala-Nissila, and S. C. Ying, Phys. Rev. E 80, 021907 (2009).

${ }^{33}$ M. McCauley, R. Forties, U. Gerland, and R. Bundschuh, Phys. Biol. 6, 036006 (2009).

${ }^{34}$ T. Sakaue, Phys. Rev. E 81, 041808 (2010).

${ }^{35}$ M. Muthukumar, J. Chem. Phys. 132, 195101 (2010).
${ }^{36}$ P. Rowghanian and A. Y. Grosberg, J. Phys. Chem. B 115, 14127 (2011).

${ }^{37}$ N. N. Jetha, C. Feehan, M. Wiggin, V. Tabard-Cossa, and A. Marziali, Biophys. J. 100, 2974 (2011).

${ }^{38}$ A. Milchev, J. Phys. Condens. Matter 23, 103101 (2011).

${ }^{39}$ M. Fyta, S. Melchionna, and S. Succi, J. Polym. Sci. Pol. Phys. 49, 985 (2011).

${ }^{40}$ C. M. Edmonds, Y. C. Hudiono, A. G. Ahmadi, P. J. Hesketh, and S. Nair, J. Chem. Phys. 136, 065105 (2012).

${ }^{41}$ N. Pizzolato, A. Fiasconaro, D. Persano Adorno, and B. Spagnolo, Phys. Biol. 7, 034001 (2010).

${ }^{42}$ A. Fiasconaro, J. J. Mazo, and F. Falo, Phys. Rev. E 82, 031803 (2010).

${ }^{43}$ A. Fiasconaro, J. J. Mazo, and F. Falo, J. Stat. Mech.: Theory Exp. (2011) P11002.

${ }^{44}$ A. Fiasconaro, J. J. Mazo, and F. Falo, New J. Phys. 14, 023004 (2012).

${ }^{45}$ J. Shin and W. Sung, J. Chem. Phys. 136, 045101 (2012).

${ }^{46}$ T. Ikonen, J. Shin, W. Sung, and T. Ala-Nissila, J. Chem. Phys. 136, 205104 (2012).

${ }^{47}$ D. Valenti, G. Denaro, D. Persano Adorno, N. Pizzolato, S. Zammito, and B. Spagnolo, Cent. Eur. J. Phys. 10(3), 560 (2012).

${ }^{48}$ C. R. Doering and J. C. Gadoua, Phys. Rev. Lett. 69, 2318 (1992).

${ }^{49}$ M. Bier and R. D. Astumian, Phys. Rev. Lett. 71, 1649 (1993).

${ }^{50}$ M. Boguna, J. M. Porra, J. Masoliver, and K. Lindenberg, Phys. Rev. E 57, 3990 (1998).

${ }^{51}$ R. N. Mantegna and B. Spagnolo, Phys. Rev. Lett. 84, 3025 (2000).

${ }^{52}$ A. A. Dubkov, N. V. Agudov, and B. Spagnolo, Phys. Rev. E 69, 061103 (2004).

${ }^{53}$ B. Spagnolo, A. A. Dubkov, A. L. Pankratov, E. V. Pankratova, A. Fiasconaro, and A. Ochab-Marcinek, Acta Phys. Pol. B 38, 1925 (2007).

${ }^{54}$ A. Fiasconaro and B. Spagnolo, Phys. Rev. E 83, 041122 (2011).

${ }^{55}$ P. E. J. Rouse, J. Chem. Phys. 21, 1272 (1953).

${ }^{56}$ J. Tothova, B. Brotovsky, and V. Lisy, Czech. J. Phys. 55, 221 (2005).

${ }^{57}$ M. Muthukumar and A. Baumgartner, Macromolecules 22, 1937 (1989).

${ }^{58}$ P. G. de Gennes, Proc. Natl. Acad. Sci. U.S.A. 96, 7262 (1999).

${ }^{59}$ W. Sung and P. J. Park, Phys. Rev. Lett. 77, 783 (1996).

${ }^{60}$ M. Muthukumar, Phys. Rev. Lett. 86, 3188 (2001).

${ }^{61}$ M. Muthukumar, J. Chem. Phys. 118, 5174 (2003).

${ }^{62}$ M. Muthukumar, Annu. Rev. Biophys. Biomol. Struct. 36, 435 (2007).

${ }^{63}$ S. E. Henrickson, M. Misakian, B. Robertson, and J. J. Kasianowicz, Phys. Rev. Lett. 85, 3057 (2000).

${ }^{64}$ S. Howorka, L. Movileanu, X. Lu, M. Magnon, S. Cheley, O. Braha, and H. Bayley, J. Am. Chem. Soc. 122, 2411 (2000).

${ }^{65}$ J. J. Kasianowicz, S. E. Henrickson, H. H. Weetall, and B. Robertson, Anal. Chem. 73, 2268 (2001).

${ }^{66}$ B. Spagnolo, S. Spezia, L. Curcio, N. Pizzolato, A. Fiasconaro, D. Valenti, P. Lo Bue, E. Peri, and S. Colazza, Eur. Phys. J. B 69, 133 (2009). 